\title{
1,3-Dichloropropenes - in the preparation of thiazole derivatives - 2-chloro-5-chloromethylthiazole and 5-hydroxymethylthiazole
}

\author{
Gregory F. Hillstrom, Michelle A. Hockman, Ramiah Murugan*, Eric F.V. Scriven, James \\ R. Stout, and Jason Yang
}

Reilly Industries, Inc., 1500 South Tibbs Ave., Indianapolis, IN 46242-0912

E-mail: rmurugan@reillyind.com

\section{Dedicated to Professor Rudy Abramovitch on the occasion of his $70^{\text {th }}$ birthday (received 21 Jun 01; accepted 11 Oct 01; published on the web 19 Oct 01)}

\begin{abstract}
1,3-Dichloropropene (a mixture of cis and trans isomers) has been used to synthesize thiazole derivatives by starting its reaction with sodium thiocyanate to give 3-chloro-2propenylthiocyanate. This thiocyanate on heating undergoes thermal [3,3]-sigmatropic rearrangement to give the isothiocyanate derivative, 3-chloro-1-propenylisothiocyanate. This mixture of cis and trans isomers of isothiocyanate on chlorination gave 2-chloro-5chloromethylthiazole. 5-Hydroxymethylthiazole is made from 2-chloro-5-chloromethyl thiazole in two steps, first by displacement of the aliphatic halogen with formate anion followed by hydrolysis of the formate ester and hydrodehalogenation of the aromatic halogen using hydrogen and palladium on carbon catalyst.
\end{abstract}

Keywords: 1,3-Dichloropropene, 2-chloro-5-chloromethylthiazole, 5-hydroxymethylthiazole, thiocyanate, isothiocyanate, chlorination

\section{Introduction}

Heterocycles based on thiazole ring systems are biologically active. Hence, thiazole ring systems containing molecules are known in pharmaceuticals as well as in agrochemical products. ${ }^{1-6}$ For example, ritonavir an anti-HIV drug contains a 5-substituted oxymethylthiazole moiety, and an isostere of the important insecticide, imidacloprid has 2-chloro-5-substituted methylthiazole as part of the molecule (Figure 1). 


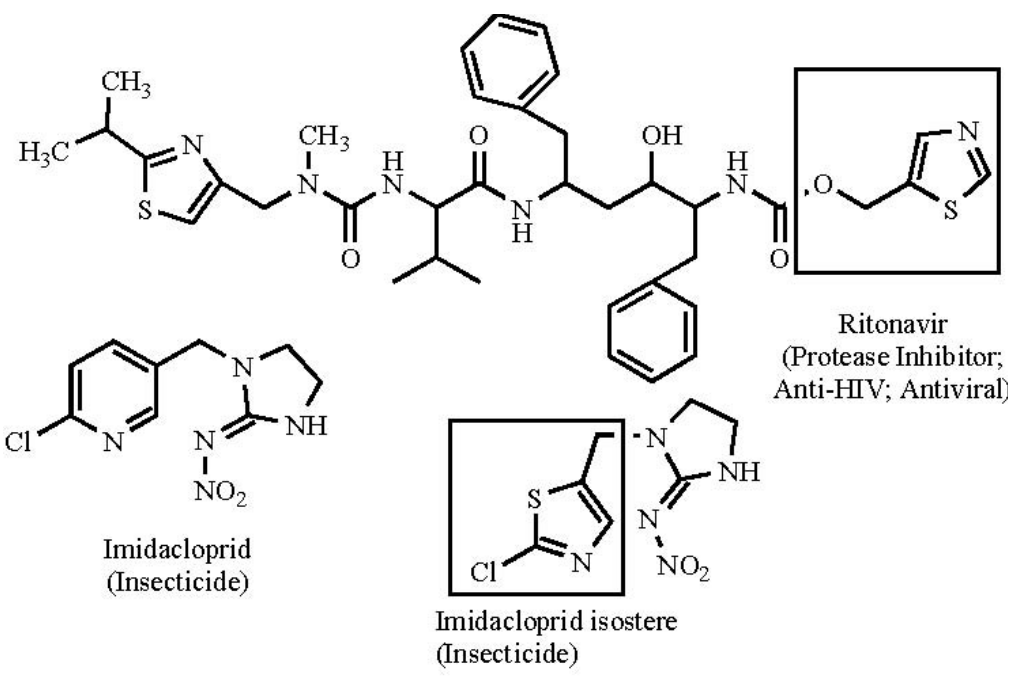

Figure 1

\section{Results and Discussion}

5-Hydroxymethylthiazole (5) has been produced in four steps starting with 1,1,3,3tetramethoxypropane (1) (a malondialdehyde synthon) (Scheme 1).
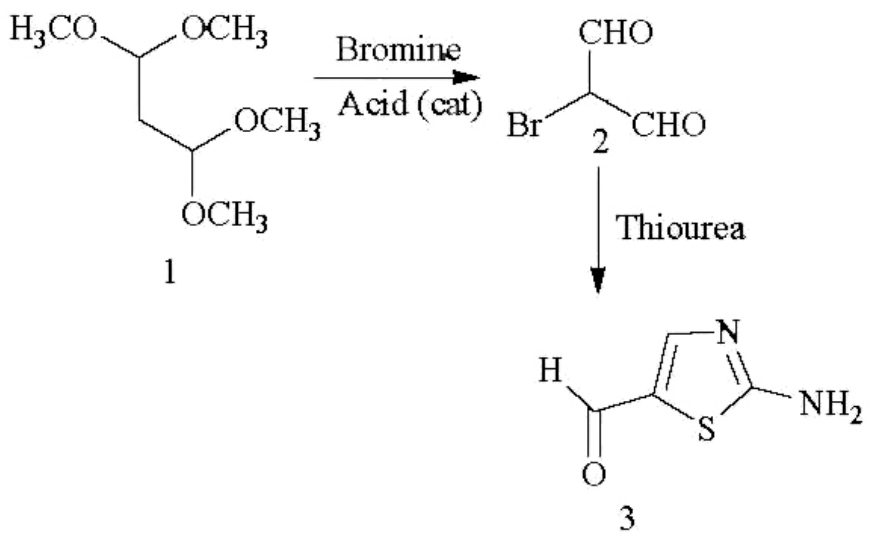

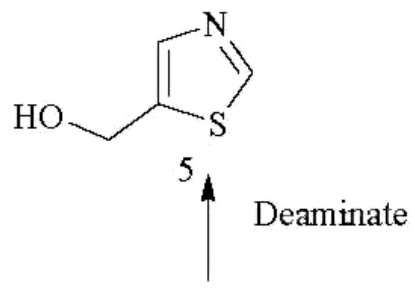

Reduce

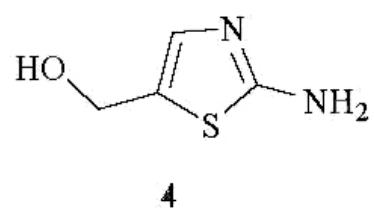

\section{Scheme 1}

The high cost of the starting material, 1,1,3,3-tetramethoxypropane (1), led us to search for an alternative method to make 5-hydroxymethylthiazole (5). During the literature search we came across the intermediate 2-chloro-5-chloromethylthiazole (10) which may be made readily from allylisothiocyanate (6) and chlorine ${ }^{1}$ or from 2-chloro-allylisothiocyanate (7) and chlorine (Scheme 2). ${ }^{2}$

From an investigation of the intermediates of the reaction between allylisothiocyanate (6) (obtained by the reaction of allyl chloride and sodium thiocyanate) and chlorine (Scheme 2) and 
2-chloroallylisothiocyanate (7) (obtained by the reaction of 2,3-dichloropropene and sodium thiocyanate) and chlorine (Scheme 2), we came to the conclusion that the reaction of 1chloroallylisothiocyanate and chlorine should give the same end product, 2-chloro-5chloromethylthiazole (10). Such an allylisothiocyanate, 1-chloro-allylisothiocyanate, could be made from the reaction of 1,3-dichloropropene (11) and sodium thiocyanate (Scheme 3 ). ${ }^{3}$

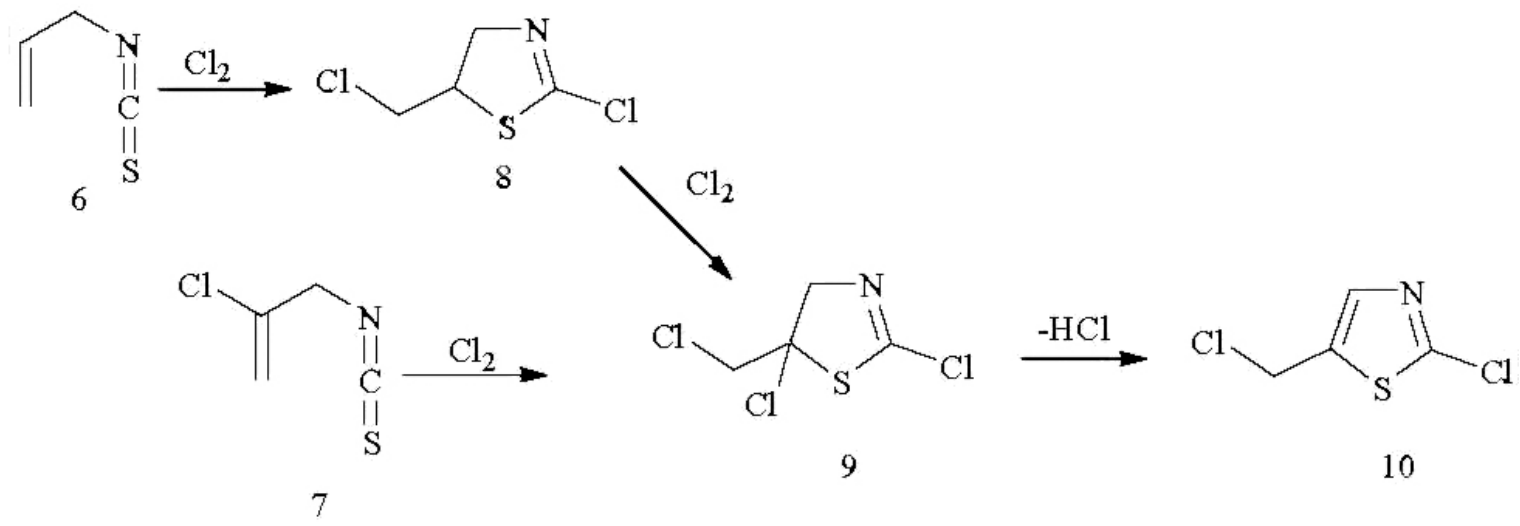

\section{Scheme 2}

The reaction of 1,3-dichloropropene (mixture of cis and trans isomers) with sodium thiocyanate initially gave the 3-chloro-2-propenylthiocyanate (12) (mixture of cis and transisomers), which underwent a [3.3]-sigmatropic rearrangement to give the 1-chloro-2propenylisothiocyanate intermediate which readily rearranged (1,3-halogen shift) to give 3chloro-1-propenylisothiocyanate (13) (mixture of cis and trans isomers). Chlorination of this mixture of isothiocyanates obtained from the reaction of 1,3-dichloropropenes and sodium thiocyanate, gave 2-chloro-5-chloromethylthiazole (10) $)^{4,5}$ (Scheme 3).<smiles>N#CCNC=CCl</smiles>

11<smiles>N#CSCC=CCl</smiles>

12

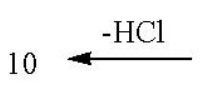<smiles>COC=CCCl</smiles>

$\mathrm{Cl}_{2}$

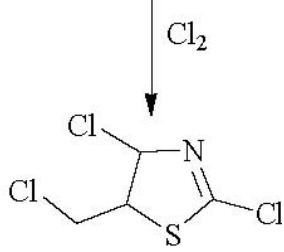

\section{Scheme 3}

The reaction of 2-chloro-5-chloromethylthiazole (10) with sodium formate followed by hydrolysis gave 2-chloro-5-hydroxymethyl-thiazole (15), which on dehalogenation with 
hydrogen and palladium on carbon catalyst gave 5-hydroxymethylthiazole (5) ${ }^{6}$ (Scheme 4).

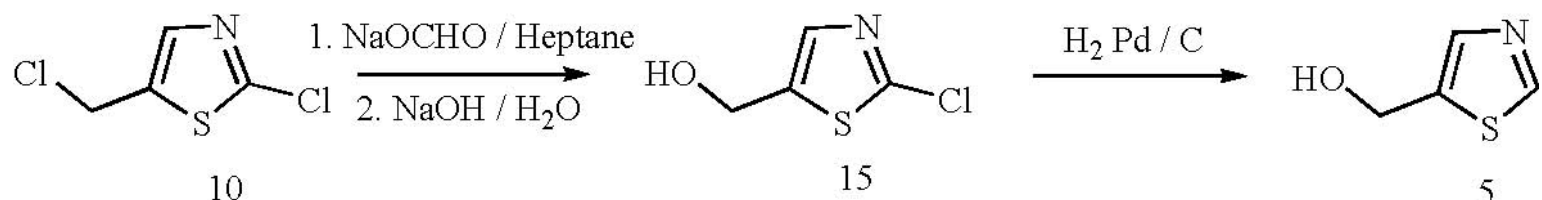

\section{Scheme 4}

\section{Summary}

Thiazole derivatives are made from 1,3-dichloropropene (a mixture of cis and trans isomers) by starting the reaction with sodium thiocyanate, which gives 3-chloro-2-propenylthiocyanate. This thiocyanate undergoes a thermal [3,3]-sigmatropic rearrangement to give the isothiocyanate derivative, 3-chloro-1-propenylisothiocyanate. This mixture of the cis and trans isomers of 3chloro-1-propenylisothiocyanates on chlorination gives 2-chloro-5-chloromethylthiazole in moderate yields. 2-Chloro-5-chloromethylthiazole is a versatile intermediate for making agrochemicals and pharmaceuticals. 5-Hydroxymethylthiazole, an intermediate to a pharmaceutical drug, is made from 2-chloro-5-chloromethyl thiazole in two steps, first by displacement of the aliphatic halogen with formate anion followed by hydrolysis of the formate ester and dehydrohalogenation of the aromatic halogen with hydrogen and a palladium catalyst.

\section{Experimental Section}

General Procedures. All the compounds mentioned in this paper are known compounds and their full characterization data have been previously reported in the literature. Confirmation of the compounds in our labs, was done using, ${ }^{1} \mathrm{H}$ NMR, ${ }^{13} \mathrm{C}$ NMR and GCMS as appropriate. The NMR spectra were recorded on a Bruker AC $300 \mathrm{MHz}$ instrument.

3-Chloro-1-isothiocyanato-1-propene ${ }^{3}$ (13). A five liter four neck flask equipped with a mechanical stirrer, thermometer and a condenser was charged with finely ground sodium thiocyanate $(401.5 \mathrm{~g}, 4.95 \mathrm{~mole})$, acetonitrile $(2000 \mathrm{~mL})$, and cis and trans 1,3-dichloro-1propene (11) (500 g, 4.51 mole). The mixture was stirred and heated to approximately $30{ }^{\circ} \mathrm{C}$ and held at this temperature for $20 \mathrm{~h}$. The precipitated sodium chloride was filtered from the mixture and the acetonitrile was removed by rotary evaporation (the concentrate contained approximately $10 \%$ of acetonitrile). The crude product was washed with water $(3 \times 350 \mathrm{~mL})$. The separated organic layer was dried over anhydrous sodium sulfate. After the sodium sulfate was filtered off, the filtrate was diluted with 1,4-dioxane $(1000 \mathrm{~mL})$ and the mixture was heated to reflux with 
stirring for $10 \mathrm{~h}$. When $\mathrm{GC}$ analysis indicated that the reaction was complete, the reaction mixture was cooled to room temperature and filtered. The filtrate was concentrated on the rotary evaporator to remove dioxane (the resulting concentrate contains approximately $10-15 \%$ of dioxane). The crude isothiocyanate product was obtained in $70 \%$ yield and used without further purification. ${ }^{1} \mathrm{H}$ NMR $\left(\mathrm{CDCl}_{3}\right) \delta$ : 5.5 to 6.3 (m, cis and trans $-\mathrm{CH}=\mathrm{CH}-$ ); $4.0 \mathrm{~d}, 4.1 \mathrm{~d}$ (cis and trans $\left.-\mathrm{CH}_{2}-\right) .{ }^{13} \mathrm{C} \mathrm{NMR}\left(\mathrm{CDCl}_{3}\right) \delta: 119.3,120.5,123.5,127.1$ (cis and trans $\left.-\mathrm{CH}=\mathrm{CH}-\right) ; 37.7$, 41.1 (cis and trans $-\mathrm{CH}_{2}$ ).

2-Chloro-5-chloromethylthiazole $\mathbf{e}^{1,2,4,5}(\mathbf{1 0})$. To a 2-liter round bottomed flask equipped with a mechanical stirrer, thermometer and a condenser, whose outlet was vented into an aqueous sodium hydroxide trap, was charged the crude mixture of cis- and trans-3-chloropropenyl isothiocyanates (13) (504.2 g, 3.79 mole ) and chloroform (600 mL). The mixture was stirred and heated to reflux. Chlorine (267.1 g, 3.77 mole) gas was bubbled under the surface of the reaction over a period of 6-8 $\mathrm{h}$. The conversion of the isothiocyanates to 2-chloro-5(chloromethyl)thiazole (10) was monitored by GC. When the reaction was shown to be complete by GC analysis, the reaction mixture was cooled and then filtered. The filtrate was concentrated on the rotary evaporator to remove chloroform. The yield of 2-chloro-5chloromethylthiazole (10) based on the weight of concentrate and GC purity was around 65\%. Sodium bicarbonate (approximately 0.25 equivalents) was added to the concentrated filtrate and the product 2-chloro-5-chloromethylthiazole (10) was distilled at $97{ }^{\circ} \mathrm{C}$ under vacuum $(6 \mathrm{~mm} / \mathrm{Hg})$. The yield after distillation was 43\%. ${ }^{1} \mathrm{H} \mathrm{NMR}\left(\mathrm{CDCl}_{3}\right) \delta: 7.3(\mathrm{~s}, 1 \mathrm{H}) ; 4.6(\mathrm{~s}, 2 \mathrm{H})$. ${ }^{13} \mathrm{C} \mathrm{NMR}\left(\mathrm{CDCl}_{3}\right) \delta: 152.6$ (C-2); 140.2 (C-4); 137.5 (C-5); $37.1\left(-\mathrm{CH}_{2}-\right)$.

2-Chloro-5-hydroxymethylthiazole ${ }^{6}$ (15). To a $500 \mathrm{~mL}$ round bottom flask equipped with mechanical stirrer, thermometer and condenser was charged with sodium formate $(121.5 \mathrm{~g}, 1.8$ mole, 3 eq.), Aliquat 336 (tricaprylylmethylammonium chloride) (4.81 g, 11.9 mmole, 0.02 eq.), and $125 \mathrm{~mL}$ heptane. The reaction was heated to reflux, 2-chloro-5-chloromethylthiazole (10) (CCMT, pre-washed with sodium bicarbonate) (100 g, 0.6 mole) was slowly added. The reaction continued at reflux overnight. GC analysis of the oily layer showed $72 \%$ starting material CCMT. The reaction was then filtered and concentrated. The recovered concentrate was then used in another reaction. In a similarly equipped flask was charged sodium formate ( 85.34 $\mathrm{g}, 1.26 \mathrm{~mole})$, Aliquat 336 (3.18 g, $7.87 \mathrm{mmole})$ and $88 \mathrm{~mL}$ heptane. The reaction was heated to reflux, and then the above recovered material $(70.24 \mathrm{~g})$ was dripped into the reaction. The reaction continued at reflux overnight. The reaction was cooled and sodium hydroxide was added (140 g of 25\% aqueous solution, made from 50\% and ice) slowly keeping the reaction temperature below $30{ }^{\circ} \mathrm{C}$. The reaction was stirred for 30 minutes. Aqueous sodium chloride $(70 \mathrm{~mL}, 20 \%)$, water $(30 \mathrm{~mL})$ and tert-butyl methyl ether $(100 \mathrm{~mL})$ were added to the reaction and stirred for 15 minutes. The organic layer separated and the aqueous portion was extracted with tert-butyl methyl ether $(3 \mathrm{X} 100 \mathrm{~mL})$, combined with the organic layer and dried over sodium sulfate. The reaction was filtered. Carbon $(6.6 \mathrm{~g})$ and silica gel (13.23 g) were added to the reaction and stirred for 30 minutes. The reaction was then filtered to remove the solids and color, more tert-butyl methyl ether used as a wash $(30 \mathrm{~mL})$. The filtrate was then concentrated. 
The yield of 2-chloro-5-hydroxymethylthiazole (15) was 40\% based on CCMT added in the first reaction. The 2-chloro-5-hydroxymethylthiazole (15) thus obtained was used without purification in the next step.

Preparation of 5-Hydroxymethylthiazole $^{6}$ (5). A 1.4 liter Parr hydrogenation shaker bomb was loaded with 2-chloro-5-hydroxymethylthiazole (15) (57.25 g, 0.38 mole), palladium on carbon (19.0 g , 5\% and 50\% water wet), sodium acetate $(36.0 \mathrm{~g})$ and methanol $(600 \mathrm{~mL})$. The bomb was sealed, pressurized to $150-200$ psi with hydrogen, and heated while shaking at $65{ }^{\circ} \mathrm{C}$ for 19 $\mathrm{h}$. After cooling and venting the bomb the contents of the bomb are poured out. The palladium on carbon catalyst was filtered off and to the filtrate toluene $(100 \mathrm{~mL})$ was added and the salts were filtered off. The filtrate was concentrated and distilled (bp $114{ }^{\circ} \mathrm{C} / 1.5 \mathrm{~mm} / \mathrm{Hg}$ ) to yield $76.8 \%$ of 5-hydroxymethylthiazole (5). ${ }^{1} \mathrm{H}$ NMR $\left(\mathrm{CDCl}_{3}\right) \delta: 8.6(\mathrm{~s}, 1 \mathrm{H}) ; 7.6(\mathrm{~s}, 1 \mathrm{H}) ; 5.2$ (bs, $1 \mathrm{H}) ; 4.8(\mathrm{~s}, 2 \mathrm{H}) .{ }^{13} \mathrm{C} \mathrm{NMR}\left(\mathrm{CDCl}_{3}\right) \delta: 153.6$ (C-2); 140.1 (C-4); 139.8 (C-5); $56.7\left(-\mathrm{CH}_{2}-\right)$.

\section{References}

1. Beck, G.; Heitzer, H. U.S. Patent 4,748,243; issued May 31, 1988; filed Aug 31, 1987; to Bayer Aktiengesellschaft.

2. Osaka, H. U1.; Matsubara, N. H.; Kawabe, I. M. U. S. Patent 5,180,833; issued Jan 19, 1993; filed Mar 7, 1991; to Takeda Chemical Industries, Ltd.

3. Schulze, K.; Richter, F.; Seisheit, R.; Krause, R.; Muhlstadt, M. J. Prakt. Chemie. 1980, 322, 629.

4. Murugan, R.; Scriven, E. F. V. PCT Int. Appl. WO 98 45,279 (Oct 15, 1998); Reilly Industries Inc., Chem. Abstr. 1998, 129, 302633v.

5. Jackson, A.; Heyes, G.; Grayson, J. I.; Clarke, R. U.S. Patent 5,705,652 (Jan 6, 1998); Fine Organics Ltd., and Agro-Kanesho Company.

6. Leanna, R. M.; Morton, H. E. PCT Int. Appl. WO 96 16,050 (May 30, 1996); Abbott Laboratories, USA. Chem. Abstr. 1996, 125, $114603 \mathrm{~d}$. 